\section{Oral Presentations: $1^{\text {st }}-3^{\text {rd }}$ June 2015}

\section{IS PRE-EXPOSURE PROPHYLAXIS FOR HIV PREVENTION COST-EFFECTIVE IN MEN WHO HAVE SEX WITH MEN WHO ENGAGE IN CONDOMLESS SEX IN THE UK?}

${ }^{1}$ Valentina Cambiano*, ${ }^{2}$ Alec Miners, ${ }^{3}$ David Dunn, ${ }^{3}$ Sheena McCormack, ${ }^{4}$ Noel Gill, ${ }^{4}$ Anthony Nardone, ${ }^{4}$ Monica Desai, ${ }^{5}$ Gus Cairns, ${ }^{1}$ Alison Rodger, ${ }^{1}$ Andrew Phillips. ${ }^{1}$ University College London, London, UK; ${ }^{2}$ London School of Hygiene and Tropical Medicine, London, UK; ${ }^{3}$ MRC Clinical Trials Unit at UCL, London, UK; ${ }^{4}$ Public Health England, London, UK; ${ }^{5}$ NAM, London, UK

\subsection{6/sextrans-2015-052126.1}

Background Pre-exposure prophylaxis (PrEP) is highly protective against sexual acquisition of HIV among men having sex with men (MSM). The cost-effectiveness of PrEP will play a major role in deciding whether the NHS should introduce PrEP. Aim To evaluate the cost-effectiveness of introducing PrEP among MSM in the UK.

Methods An individual-based dynamic stochastic model calibrated to the HIV epidemic among MSM in the UK was used. It was assumed that, from 2016, 50\% of people who tested negative for HIV and who had periods of condomless sex with a long-term or casual partners would use PrEP during such periods. While on PrEP men would be tested three-monthly and PrEP discontinued if diagnosed HIV-positive or if not engaging in condomless sex for that three-month period (and restarting PrEP if again engaging in condomless sex).

Results Preliminary results indicate that the introduction of PrEP would lead to a gain in quality-adjusted life years. If current costs of antiretrovirals and PrEP are assumed for the next 30 years (as is generally regarded as good practice in the base cases analysis) PrEP introduction is not cost-effective. However, when considering likely reductions in costs of antiretrovirals and PrEP, due to the use of generic drugs, PrEP would likely be costeffective.

Conclusion Our preliminary evaluation suggests that the use of PrEP for MSM during periods of condomless sex is not cost effective at current antiretroviral prices, but it would become cost-effective if drug prices are reduced after patent expiry date.

\section{AN EPIDEMIOLOGICAL ANALYSIS OF MEN WHO HAVE SEX WITH MEN (MSM) WHO ARE PRESCRIBED HIV POST-EXPOSURE PROPHYLAXIS: IMPLICATIONS FOR WIDER PRE-EXPOSURE PROPHYLAXIS POLICY}

${ }^{1}$ Holly Mitchell ${ }^{*},{ }^{1}$ Martina Furegato, ${ }^{1}$ Gwenda Hughes, ${ }^{1,2}$ Nigel Field, ${ }^{1}$ Hamish Mohammed, ${ }^{1}$ Anthony Nardone. 'Public Health England, London, UK; ${ }^{2}$ University College London, London, UK

\section{$10.1136 /$ sextrans-2015-052126.2}

Background/introduction Post-exposure prophylaxis following sexual exposure (PEPSE) is a potential method of preventing HIV infection in certain circumstances. Initiation of PEPSE is recommended following receptive anal intercourse with a partner of known positive or unknown HIV status from a high risk group.

Aim(s)/objectives To investigate the characteristics and risk profile of patients receiving PEPSE to determine whether this could inform development of pre-exposure prophylaxis (PrEP) policy for men who have sex with men (MSM).

Methods Data from the Genitourinary Medicine Clinic Activity Dataset (GUMCADv2) were used to investigate the characteristics of patients receiving PEPSE. Associations with PEPSE use were assessed using multivariate logistic regression.

Results Between 2011 and 2013, 14,118 patients received PEPSE, of which 63\% $(8,896)$ were MSM. Among MSM receiving PEPSE, $14 \%(1,213)$ received more than one course (maximum 13 courses), 45\% $(3,990)$ were aged $25-34$ years and $75 \%$ $(6,702)$ were of white ethnicity. $2.0 \%$ were diagnosed with HIV between 4 and 16 months after receiving their last course of PEPSE. Compared to MSM controls not receiving PEPSE, MSM receiving PEPSE were significantly more likely to be of nonwhite ethnicity (adjusted OR $=1.28,95 \%$ CI 1.21-1.36), and to be diagnosed with HIV following a subsequent exposure (adjusted OR $=1.21,95 \%$ CI 1.03-1.41).

Discussion/conclusion MSM prescribed PEPSE are at high risk of acquiring HIV infection following a subsequent exposure and may require intensive interventions to ensure course completion and reduce HIV risk behaviour. PrEP may be beneficial for highrisk MSM receiving PEPSE and also avoid the need for repeat PEPSE prescriptions.

\section{IS POINT OF CARE TESTING 'UNSAFE IN THE CITY'?}

Susanna Currie*, Debbie Thomas, Alma Hatley, Sean Rezvani, Orla McQuillan. Manchester Royal Infirmary, Manchester, UK

10.1136/sextrans-2015-052126.3

Background Point of care testing (POCT) for HIV is acknowledged in UK Guidelines as useful outside clinic settings, but is it safe? Our 10 year POCT programme has experienced false positives and negatives which resulted in our use of back up serology samples as standard; this differs from practice within POCT elsewhere. We had a televised false positive POCT result on "Unsafe Sex in the City" and 4 false positives in a year which caused a temporary shutdown of our POCT programme and an MRHA investigation.

Aim To review the need for back-up serology with POCT.

Method A retrospective review of all Alere Determine ${ }^{\mathrm{TM}}$ HIV-1/ $2 \mathrm{Ag} / \mathrm{Ab}$ Combo tests at a City Centre outreach service in 2013. Results were compared with concomitant serology.

Results POCT was provided for 382 patients. Three patients declined POCT; 2 POCT results were not documented; 10 did not have serology in parallel.

Of the remaining 367 patients: 3 true positives $(0.8 \%) ; 2$ false positives (0.6\%); and 3 false negatives $(0.8 \%)$. Negative predictive value 99.2\%; Positive predictive value 60\%; Sensitivity $50 \%$; Specificity $99.4 \%$.

Discussion This is data providing statistics for POCT in real time. Compared to advertised values Alere is underperforming. The negative predictive value is reassuring; however, the sensitivity of the test is unacceptable. Had 3 of our patients not had back up serology, they would have been unaware of their diagnosis, receiving false reassurance and potentially causing unintentional HIV transmission. Do we take this risk on board and perform POCT without back up serology? 